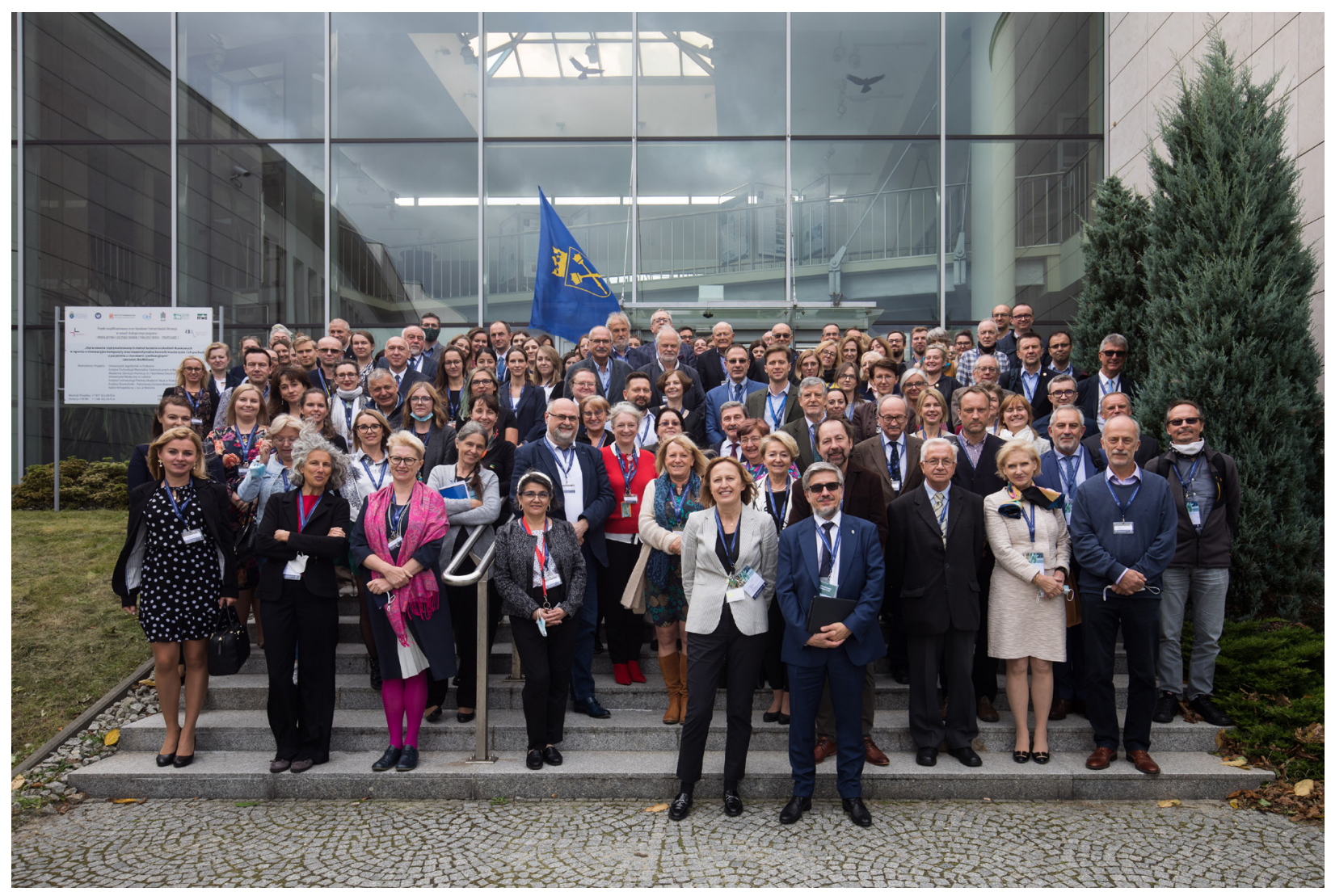

Participants on the 2nd day of Conference. Phot. Jakub Włodek.

\title{
The Jubilee Conference entitled: The latest achievements in biochemistry, biophysics and biotechnology - 50 years of history of the Faculty of Biochemistry, Biophysics and Biotechnology of the Jagiellonian University in Kraków
}

The Jubilee Conference of the Faculty of Biochemistry, Biophysics and Biotechnology of the Jagiellonian University (FBBB JU) was held in Kraków on September 23-24, 2021. The honorary patronage of the Conference was given by Professor Jacek Popiel, Rector of the Jagiellonian University.

The Jubilee Conference was the main event related to the celebration of the 50th anniversary of the establishment of the Institute of Molecular Biology of the Jagiellonian University (IMB JU). The IBM UJ, created in 1970, originally consisted of five departments and had grown for the next 30 years, significantly increasing in the staff and infrastructure. After moving to a new building at its current location in 2001, IMB JU was transformed into Faculty of Biotechnology in 2002, and in 2006 this Faculty has changed its name to the Faculty of Biochemistry, Biophysics, and Biotechnology. At present, FBBB JU consists of 15 Departments and 2 independent Laboratories, and employs over 250 people.

Over 370 participants took part in the event, 270 of whom participated on-site, and the rest participated remotely via the Microsoft Teams platform. Representatives of the Jagiellonian University authorities, as well as of leading scientific centres from Poland were invited to the Faculty. The programme of the Conference consisted of 2 parts. The first one was mainly devoted to the history of the Faculty, and the second one was strictly scientific.

The official part of day 1 was opened by prof. Józef Dulak, the Chair of the Organizing Committee, followed by the Dean - prof. Jolanta Jura, and by prof. Piotr Kuśtrowski, the Vice-Rector for scientific research. This part was followed by presentations delivered by previous Deans of the Faculty - Prof. Wojciech Froncisz, Kazimierz Strzal$\mathrm{ka}$ and Zbigniew Madeja. The first part of the conference was culminated by congratulations, best wishes and gifts to the Faculty and the Dean, from prof. Ewa Lojkowska, Chairwoman of the Committee for Biotechnology, PAN, dr. Monika Liguz-Lęcznar, Vice-Director for Scientific Affairs of the Marceli Nencki Institute of Experimental Biology PAN, and prof. Andrzej Legocki, Chair of the Polish Biochemical Society. 
The scientific part of the Conference (see https://jubileuszwbbib.confer.uj.edu.pl/en_GB/program) began with a plenary lecture by professor Virginijus Šikšnys from the University of Vilnus, devoted to the way of action of molecular tools serving to edit DNA - CRISPR-Cas9. Prof. Šikšnys is one of the discoverers of this system as the universal method of gene editing, for which he was awarded numerous honors, including the 2018 Kavli Prize in Nanotechnology.

On the second day of the meeting, 17 more talks were delivered. The presentations were divided into five sessions of variable but very up-to-date topics, representing the research field of FBBB UJ.

The first four lectures concerned the molecular and cytologic aspects of cancerogenesis and cancer treatment. The second session concerned the nervous system and neurons, and the third session was focused on virus and prion diseases, including COVID 19 and the Creutzfeldt-Jacob disease. The fourth session was devoted to etiology and treatment of periodontitis, followed by a session concerning the phenomena of electron transfer in the respiratory chain and photosynthesis, mitochondrial-derived diseases, and micro-RNA.

The Jubilee Conference turned out to be a great scientific and social success. The lectures were at a high scientific level, and the historical session allowed to realize all the phenomena which lead to the present position of the Faculty. It also gave the opportunity to meet old acquaintances and establish new areas of collaboration in the future.

The Conference was vastly supported by the Ministry of Education and Science with funding from "The Excellent Science" programme.

As active participation in the lectures during the Conference was possible only for invited speakers, in order to give a chance to all participants to share their research in public, everyone was invited to submit manuscripts to a special issue of Acta Biochimica Polonica. Five researchers from the Faculty took advantage of this opportunity, and their papers are published in the present issue of ABP. The scope of these papers is wide and well reflects the research field of the Faculty. Dr Gregor Becker represents the interdisciplinary field of bioethics and presents the story of cloning Dolly, Prof. Anna Pawlak (Burakowska et al., 2021) shares the results of studying photodynamic properties of various derivatives of cholesterol towards lipids constituting biological membranes, and prof. Jarosław Czyż and colleagues propose Lactarius deterrimus, a wild-growing fungus as a new drug against gastric cancer (Król et al., 2021). This paper contains the legacy of prematurely deceased dr Damian Ryszawy from the Department of Cell Biology. Prof. Agnieszka Wolnicka-Głubisz (Linowiecka et al., 2021) analyzes the possibility of vitamin D derivatives' applications in breast cancer therapy. Finally, prof. Andrzej Kozik's group (Satala et al., 2021) demonstrates adhesive properties of an opportunistic fungal parasite, Candida albicans, related to a newly discovered adhesin - the phosphoglycerate mutase.

On behalf of the Organizing Committee,

Przemysław M. Płonka

Józef Dulak 